\title{
Metallic Foreign Bodies in the Thoracic Wall in Three Cases
}

\author{
Huseyin Fatih Sezer ${ }^{1}$ (D), Hakan Dayanir ${ }^{2}$ (D) \\ 'Department of Thoracic Surgery, Nyala Sudan Turkey Training and Research Hospital, Nyala, Sudan \\ ${ }^{2}$ Department of Anashtesia and Reanimation, Nyala Sudan Turkey Training and Research Hospital, Nyala, Sudan
}

Cite this article as: Sezer HF, Dayanir H. Metallic Foreign Bodies in the Thoracic Wall in Three Cases. Eurasian J EmergMed. 2018 ; 17 (4): $202-4$.

\begin{abstract}
There are many reports and reviews about foreign objects in various body parts, but the thoracic wall foreign objects are rarely seen, and there is no treatment consensus. In this study, we aimed to investigate the treatment of three patients who were admitted to the emergency department due to a thoracic gunshot wound. In their follow-up, they survived with a bullet in their thoracic wall, and there were no further thoracic complications associated. Details of the hospital records of the three patients referred to the Nyala Sudan-Turkey Training and Research Hospital, with foreign bodies-bullets or shrapnel-in the thoracic wall were reviewed, retrospectively. The treatment of thoracic wall foreign bodies is still controversial and as a result, small, noninfectious, noncomplicated thoracic wall foreign bodies can be followed, after consulting with the patients.
\end{abstract}

Keywords: Metallic foreign body, chest wall, three patients

\section{Introduction}

There are a great number of various reports and reviews about foreign objects in every part of the body, but thoracic wall foreign bodies are rarely described, and there is no treatment consensus (1). It is difficult to determine the location of metallic foreign bodies in the thoracic wall, and they vary with interventions and may cause complications such as pneumothorax or an internal organ injury (2). In this study, we aimed to investigate the treatment of three patients admitted to the emergency department due to a thoracic gunshot wound. In their follow-up, they survived with a bullet in their thoracic wall, and there were no further associated thoracic complications.

\section{Case Presentation}

Details of the hospital records of the three patients referred to the Nyala Sudan-Turkey Training and Research Hospital who were injured with foreign bodies-bullets or shrapnel-in the thoracic wall were retrospectively reviewed.
Case 1: A 19-year-old male was admitted a day after the firearm injury. On the physical examination performed in the previous health center, there was a suture line, approximately $2 \mathrm{~cm}$ proximally to the superior section of the left scapula and parallel to the upper border of the scapula, with a size of approximately $4 \mathrm{~cm}$ in the midclavicular area. Approximately the left 5thintercostal space (ICA)level had mid-axillary line on tube thoracostomy; there was no air leak, and also there was an oscillation, and no hemorrhagic drainage was evident. Clinical and vital findings were stable. Respiratory sounds were natural, and laboratory test results including hemogram, biochemistry analysis, and coagulation parameters were normal. A foreign body formation with metallic density was observed at the 3rd ICA level, adjacent to the intercostal muscle, between the scapula and the thoracic wall on the thoracic computed tomography (CT) and chest X-ray (Figure 1a, 1b). The patient was admitted to the service for further follow-up. On the 2nd day, the tube thoracostomy was terminated without the removal of the bullet core, and both the lung wings were seen as expansive in the control films, and the patient was discharged on the 3rd day. No complications have been encountered in further assessments.

ORCID IDs of the authors: H.F.S. 0000-0001-5812-7088; H.D. 0000-0003-2018-5818. 

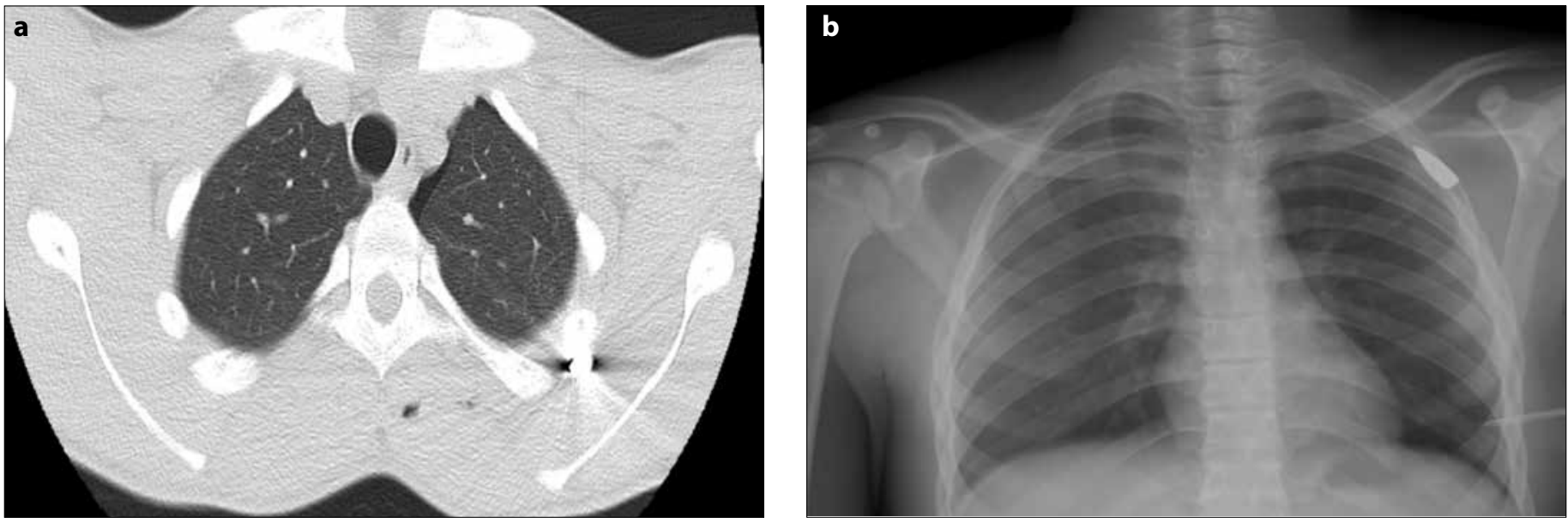

Figure 1. a, b. Computed tomography scan (a), posterior anterior chest X-ray (b)

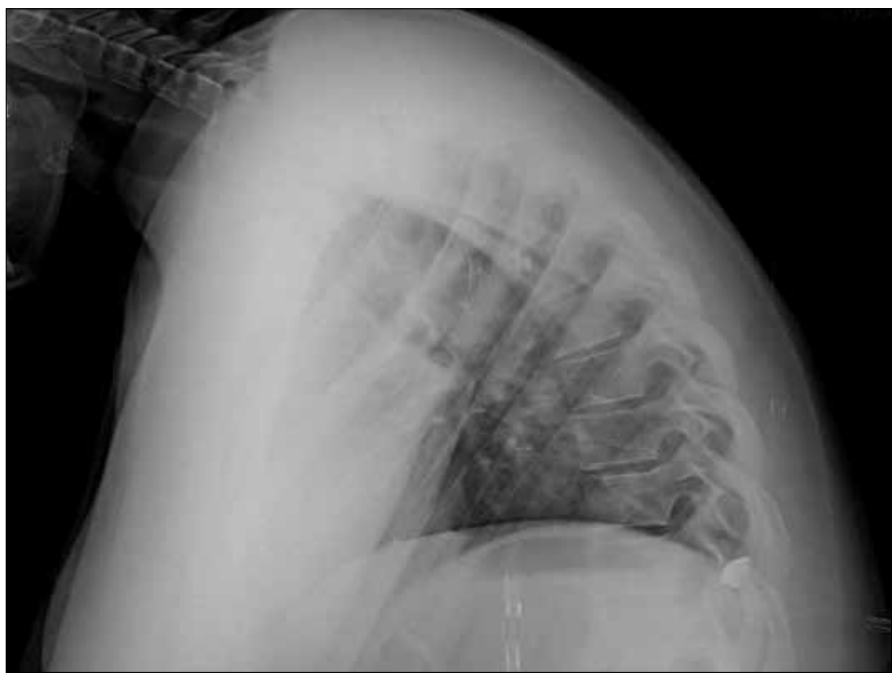

Figure 2. Lateral chest X-ray

Case 2: A 36-year-old female applied with a gunshot wound on the day of the incident. On the physical examination, a bullet entry hole approximately $1 \times 1.5 \mathrm{~cm}$ size was found in the midclavicular line at approximately the 9th-10th ICA level on the right side of the back. Clinical and vital findings were stable. Respiratory sounds were natural, and laboratory test results including hemogram, biochemistry analysis, and coagulation parameters were normal. On the chest x-ray, at the 9th-10th ICA level, the right paravertebral area, there was a foreign body formation with metallic density in the posterior thoracic wall (Figure 2), and no thoracic pathology was observed. The bullet core was not removed. The patient was followed for 1day at the hospital and discharged subsequently. No complications have been encountered in the following assessments.

Case 3: A 25-year-old male was admitted 3 days after a gunshot injury. On the physical examination, at approximately the 6th-7th ICA level, an entry gun bullet hole $1 \times 1.5 \mathrm{~cm}$ in size was present in the lateral line on the right side, and part of the bullet in the right axilla could be identified by deep palpitation. Clinical and vital findings were stable. There was sensitivity at palpation on the left side and diminished breathing sounds by auscultation. Laboratory test results including hemogram, biochemistry analysis, and coagulation parameters were normal. In the chest X-ray, there was pneumothorax on the left and in the right axillary region, a foreign body formation with metallic density at approximately the 5th-6th ICA level (Figure $3 a, 3 b)$. From the left 5th ICA mid-axillary line, tube thoracostomy was applied. The foreign body was not removed. On the 5th day, thoracic drain was terminated, and patient was discharged on the 7th day. No complications have been encountered in the next sequent assessment.

\section{Discussion}

Metallic foreign bodies in the thoracic wall are rare and are usually related to a history of trauma. Foreign bodies in the thoracic wall are more noticeable on chest radiographs and thoracic CT than on materials such as non-radio-opaque wood and plastics due to their characteristics (3). Thoracic CT provides more detailed information about characteristics of the foreign body, complete localization, other thoracic pathologies, as well as diagnosis (4). We used the plain lateral chest X-ray in three patients and thoracic CT in one patient. Verbal informed consent was obtained from patients who participated in this study.

Although the thoracic wall metallic foreign bodies do not cause any complications, they may migrate over time. They may cause pneumothorax or hemothorax, chest pain, infection, and systemic poisoning and may require further evaluating methods such as magnetic resonance imaging $(3,5)$. There are also studies in the literature reporting that metallic foreign bodies in the chest may cause hypovolemic shock due to patient's bleeding or may impair psychology of the affected person $(4,5)$. In two of our patients, we did not encounter any complications in the follow-up. One patient had unilateral pneumothorax at the time of admission and had developed hemithoraxduring tube thoracostomy. The patient's tubethoracostomy was terminated on the $2^{\text {nd }}$ day when the pneumothorax completely disappeared, and the air leak was cut off, and no complications were encountered in ongoing assessments of this patient.

The treatment of thoracic wall foreign bodies is still controversial (1). They can be followed or surgically removed. The ones that are large, sharp, infected, close to major vascular structures and may cause bleeding, loss of function, and possible intoxication 

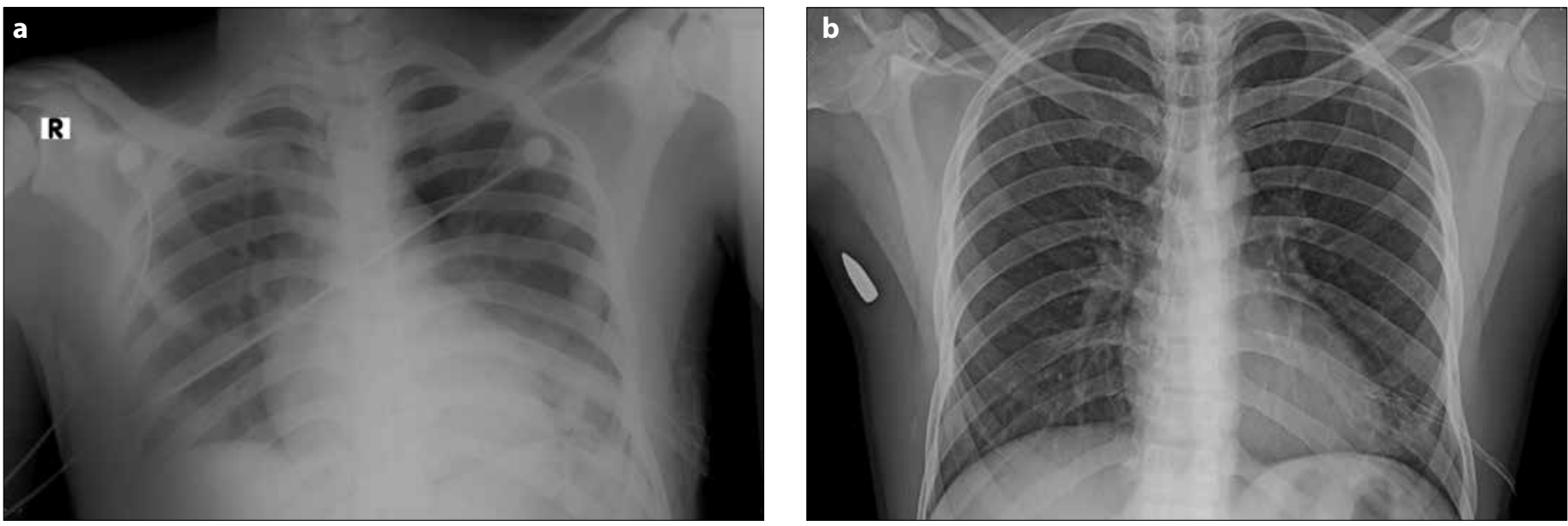

Figure 3. a, b. Posterior anterior chest X-ray with tube thoracostomy (a), posterior anterior chest X-ray after tube thoracostomy (b)

should be removed. The foreign bodies planned to be removed by surgical intervention may be found by using dynamic radiological examinations such as fluoroscopy, even if the foreign body migrates during the intervention, and thus simplifying both the surgeon's job and reducing unnecessary tissue disruption and morbidity (2). In particular, there are studies advocating the follow-up of small, non infectious, tolerated metallic foreign bodies on the chest wall that have not caused organ damage, as well as studies suggesting the removal of foreign bodies that sharp, pointed, large, contaminant and may cause hemorrhage $(1,2)$. We shared detailed information about the clinical conditions and treatment options of thoracic wall foreign bodies and the follow-up processes with our patients.

\section{Conclusion}

As a result, thoracic wall metallic foreign bodies that are small, noninfectious, blunt, uncomplicated and may not cause intoxication, bleeding, or functional disorders can be followed with a proper cooperation between the physician and the patient.

Informed Consent: Verbal informed consent was obtained from patients who participated in this study.

Peer-review: Externally peer-reviewed.
Author Contributions: Concept - H.F.S., H.D.; Design - H.F.S., H.D.; Supervision - H.F.S., H.D.; Resources - H.F.S., H.D.; Materials - H.F.S., H.D.; Data Collection and/or Processing - H.F.S., H.D.; Analysis and/or Interpretation - H.F.S., H.D.; Literature Search - H.F.S., H.D.; Writing Manuscript - H.F.S., H.D.; Critical Review - H.F.S., H.D.; Other - H.F.S., H.D.

Conflict of Interest: The authors have no conflict of interest to declare.

Financial Disclosure: The authors declared that this study has received no financial support.

\section{References}

1. Weissberg $D$, Weissberg-Kasav D. Foreignbodies in pleuraandchestwall. Ann Thorac Surg. 2008; 86: 958-61. [CrossRef]

2. Akcam Ti, Cakan A, Ergonul AG, Erol Y, Cagırıcı U. Removal of a foreign body fromthechestwall guidedbyfluoroscopy. Ege Journal of Medicine. 2013; 52: 208-10.

3. Akkas Y, Yazici UE, Aydin E. Metallic foreign body migrating to the chest wall. J Case Rep Images Surg. 2016; 2: 88-91.

4. Chang CC, LIn HJ, Foo NP, Chen KT. The hidden devil: unexpected retained knife in the chest wall. Ulus Travma Acil Cerrahi Derg. 2012; 18: 453-4. [CrossRef]

5. Karakuş A, Gungor A, Damgacı K, Kandiş H, Apaydın OO. Foreign body without complication in the thorax (bulletcore): Case report. Eurasian $J$ Emerg Med. 2011; 10: 138-40. 